
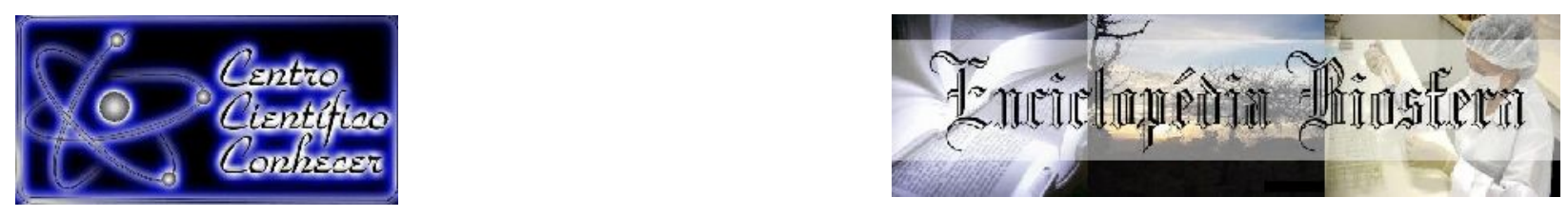

\title{
ALTERAÇÕES DE PAISAGEM NO MUNICÍPIO DE SÃO FÉLIX DO XINGU: UM ESTUDO ABORDANDO OS IMPACTOS DOS FATORES ANTRÓPICOS ENTRE 1985 A 2015
}

Ariadne Reinaldo Trindade ${ }^{1}$; Jefferson Inayan de Oliveira Souto ${ }^{2}$; Norma Ely Santos Beltrão ${ }^{3}$

${ }^{1}$ Mestranda do Programa de Pós-Graduação em Ciências Ambientais, Universidade do Estado do Pará, Belém - PA, Brasil. (dnetrindade@gmail.com).

${ }^{2}$ Mestrando do Programa de Pós-Graduação em Ciências Ambientais, Universidade do Estado do Pará, Belém - PA, Brasil.

${ }^{3}$ Docente do Programa de Pós-Graduação em Ciências Ambientais, Universidade do

Estado do Pará, Belém - PA, Brasil.

Recebido em: 06/04/2019 - Aprovado em: 10/06/2019 - Publicado em: 30/06/2019 DOI: 10.18677/EnciBio_2019A134

Mudanças no uso da terra em regiões tropicais tornaram-se mais frequentes nas últimas décadas, ocasionando intensas alterações ambientais e climáticas. 0 objetivo geral do estudo é analisar os padrões espaciais do desmatamento e temporais das variáveis climáticas (precipitação, temperatura máxima e mínima) associando com o uso e mudança da terra nos anos de 1985, 1995, 2005 e 2015 no município de São Félix do Xingu, estado do Pará. Utilizou-se um banco de dados de variáveis meteorológicas interpoladas desenvolvida pelo CLIMA, além da plataforma Google Earth Engine para gerar os valores do Índice de Vegetação por Diferença Normalizada (NDVI) e uso e mudança do solo utilizando o MapBiomas. Os resultados indicam que o município de São Félix do Xingu possui uma área territorial parcialmente antropizada nos últimos anos, quando comparado com as primeiras décadas do estudo. Além disso, considerando a influência do número crescente de áreas com pastagem, os maiores picos de temperatura máxima e mínima ocorreram principalmente entre os anos de maiores taxas de desmatamento, explicando em parte, a variabilidade anual das condições de tempo vigente pela influência de fatores antrópicos. Adicionalmente, as áreas com um número elevado de NDVI $(>0.7)$, foram parcialmente reduzidas $(<0.6)$ por causa da intensa mudança de paisagem que o munícipio de São Félix sofreu entre 2005 a 2015.

PALAVRAS-CHAVE: Amazônia, Google Earth Engine, Mudança de paisagem.

\section{LANDSCAPE CHANGES IN THE MUNICIPALITY OF SÃO FÉLIX DO XINGU: A STUDY ADDRESSING THE IMPACTS OF ANTHROPIC FACTORS BETWEEN 1985 TO 2015}

\begin{abstract}
Changes in land use in tropical regions have become more frequent in recent decades, causing intense environmental and climatic changes. The general objective of the study is to analyze the deforestation and temporal patterns of climatic variables
\end{abstract}


(precipitation, maximum and minimum temperature) associated with land use and change in 1985, 1995, 2005 and 2015 in the municipality of São Félix do Xingu, State of Pará. A database of interpolated meteorological variables developed by the CLIMA was used, as well as the Google Earth Engine platform to generate the values of the Vegetation Index by Normalized Difference (NDVI) and use and change of the soil using the MapBiomas. The results indicate that the municipality of São Félix do Xingu has a territorial area partially anthropized in recent years, when compared to the first decades of the study. In addition, considering the influence of the increasing number of pasture areas, the highest maximum and minimum temperature peaks occurred mainly between the years of higher deforestation rates, explaining in part the annual variability of the current weather conditions due to the influence of anthropic factors. In addition, areas with a high number of NDVI $(>0.6)$ were partially reduced $(<0.6)$ because of the intense landscape change that the municipality of São Félix suffered between 2005 and 2015.

KEYWORDS: Amazon, Google Earth Engine, Landscape change

\section{INTRODUÇÃO}

Entre os fatores que podem ocasionar as alterações de clima na região da Amazônia, estão as mudanças no padrão de uso do solo cujo interesse tem sido crescente tanto por estudos científicos para elucidar os impactos em um cenário de mudanças climáticas (ZEMP et al., 2017; SILVA et al., 2018), como para o planejamento de melhores políticas públicas voltadas para a região.

Observa-se, neste contexto, as preocupações referentes ao desmatamento e seus processos associados, os quais são capazes de impactar diretamente a qualidade e quantidade de recursos naturais e a saúde dos ecossistemas (AYALA et al., 2016) e clima (SPRACKLEN; GARCIA-CARRERAS, 2015), incluindo efeitos como a perda da biodiversidade, o aumento da temperatura e a redução de chuvas, tornando os ecossistemas mais sensíveis (SEDDON et al., 2016; GRIFFITHS et al., 2018).

No entanto, em muitas regiões, o impacto pela perda de florestas relacionado ao desmatamento pode ser de magnitude semelhante aos efeitos de aquecimento dos gases de efeito estufa (PIELKE et al., 2016). Com a ausência da cobertura florestal, os processos que ocorrem sobre a superfície terrestre são alterados, incluindo menores taxas de evapotranspiração, as quais afetam as condições de tempo vigente (LEJEUNE et al., 2014; DEVARAJU et al., 2015; DEBORTOLI et al., 2016).

Em regiões tropicais, a proporção de calor latente e sensível é equilibrada por extensas áreas florestais, que são essenciais na manutenção do ciclo hidrológico, formação de nuvens e precipitação (SYKTUS; MCALPINE, 2016). Todavia, nas últimas décadas, os impactos de origem antrópica, como a mudança do uso da terra na Amazônia e em outras regiões tropicais, vêm se tornando uma preocupação de âmbito global (HANSEN et al., 2013, SENIOR et al., 2017; SCOTT et al., 2018).

No Estado do Pará, as áreas de supressão vegetal que deram espaço aos pastos têm um albedo mais elevado e menor contribuição dos processos biofísicos da vegetação (SOARES-FILHO et al., 2013; STICKLER et al., 2013; SWANN et al., 2015). Menos vegetação significa um aumento gradativo da temperatura próxima à superfície terrestre (JOETZJER et al., 2013; BOISER et al., 2015; LAWRENCE; VANDECAR, 2015). 
Nessa perspectiva, o objetivo desse trabalho é analisar as mudanças de paisagem e sua influência na vegetação e clima observados no município de São Félix do Xingu em diferentes períodos, considerando os fatores de origem antrópica.

\section{Área de estudo}

\section{MATERIAIS E MÉTODOS}

A área de estudo selecionada neste trabalho, é o município de São Félix do Xingu, nas coordenadas (53ำ' W a 5051 W; 960 S a 580' S), no sudeste do estado do Pará, Brasil. Conforme a Figura 1. O município possui uma população de 91.340 pessoas e uma área territorial de $84.212,932 \mathrm{~km}^{2}$, com $22,6 \%$ de arborização de suas vias públicas (IBGE, 2017).

De acordo com Schmink et al. 2019 apesar do município ter tido no passado uma perda anual de mais de $1000 \mathrm{~km}^{2}$ de floresta, este mudou o discurso político, passando de uma visão do uso da terra mais produtivo com o gado, para a valorização das florestas como fonte de serviços ambientais. A precipitação média e a temperatura foram de $2100 \mathrm{~mm}$ e $26,5 \stackrel{\circ}{\circ} \mathrm{C}$, respectivamente. De acordo com a classificação de Koppen, o clima da região é Aw (tropical de savana com estação seca de inverno).
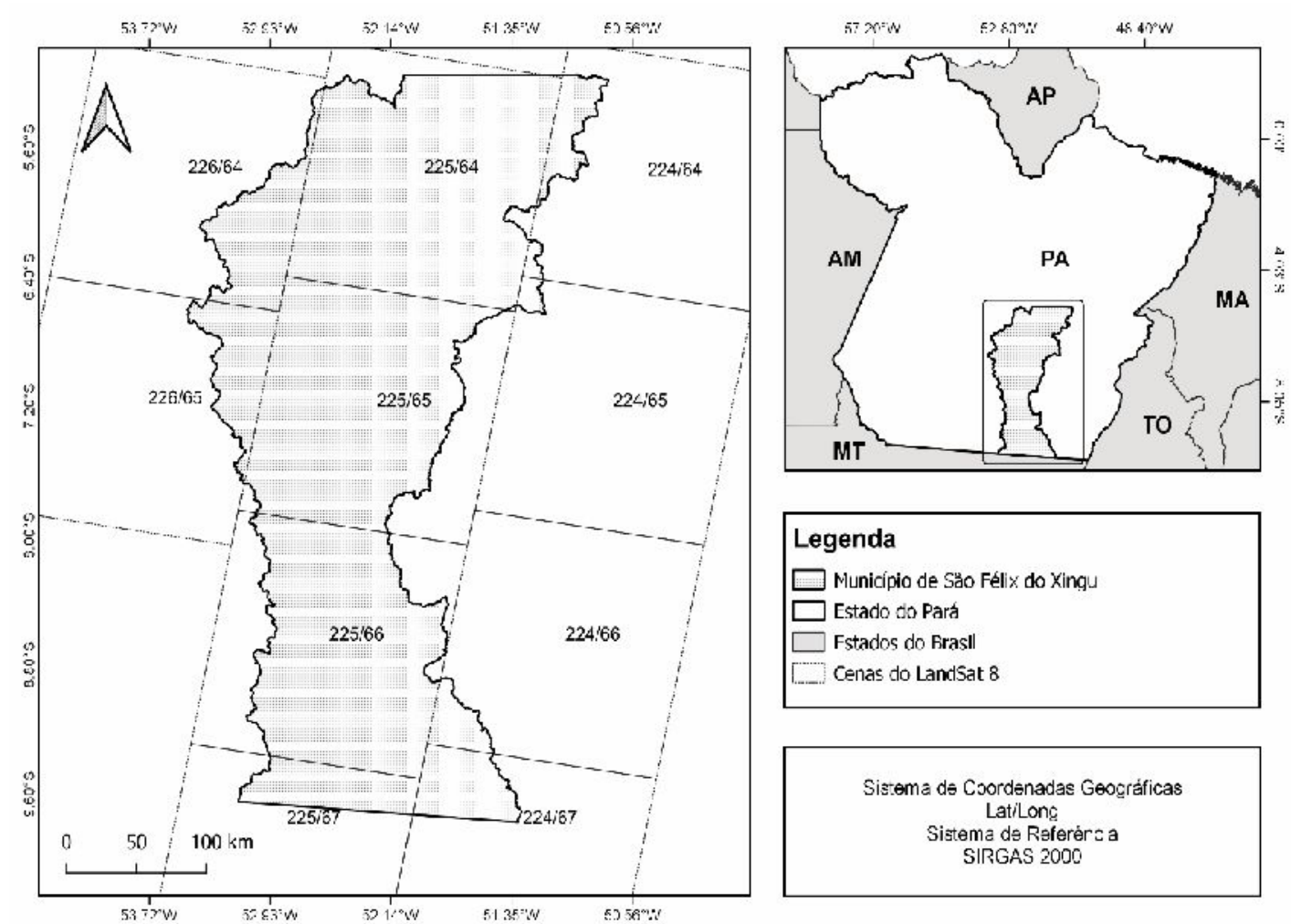

FIGURA 1 - Área de estudo: município de São Félix do Xingu, Estado do Pará.

FONTE: Autores.

\section{Variáveis meteorológicas}

Os dados de precipitação $(\mathrm{mm})$, temperatura máxima $\left({ }^{\circ} \mathrm{C}\right)$ e mínima $\left({ }^{\circ} \mathrm{C}\right)$ utilizados neste estudo, foram elaborados por Xavier et al. (2016) para todo o Brasil. Os dados foram obtidos a partir de 3625 pluviômetros e 735 estações meteorológicas para o período de 1980 a 2015, sendo que em cada variável foi escolhido o melhor método de interpolação a partir da validação cruzada. Os dados pluviométricos foram fornecidos pela Agência Nacional de Águas (ANA), pelo 
Instituto Nacional de Meteorologia (INMET) e pelo Departamento de Águas e Energia Elétrica do Estado de São Paulo (DAAE), e está disponível online em: https://utexas.app.box.com/v/xavier-etal-ijoc-data.

\section{Google Earth Engine}

O Google Earth Engine (GEE) é uma plataforma baseada em nuvem para análise geoespacial em escala planetária que traz as capacidades computacionais do Google para lidar com uma variedade de questões sociais de alto impacto (GORELICK et al., 2017), que incluem desmatamento, seca, desastres, monitoramento climático, proteção ambiental entre outros. Sendo um serviço de computação acessado e controlado por meio de uma interface de programação de aplicativo (API) acessível pela Internet e por um ambiente de desenvolvimento interativo (IDE) associado à Web que permite a criação de protótipos e a visualização rápida dos resultados, foi possível fazer uso de uma abordagem que demanda grande processamento para geração de uma composição que facilitasse a identificação e mapeamento da vegetação e uso e cobertura da terra.

As imagens baixadas e processadas no GEE, foram dos satélites Landsat 5 (1985/1995), Landsat 7 (2005) e Landsat 8 (2017). O Landsat 5 foi lançado em 1984 e registra dados em 7 bandas espectrais, com resolução espacial de $30 \mathrm{~m}$ e resolução temporal de 16 dias. O Landsat 7, foi lançado em 1999, registrando dados em 8 bandas, com resolução espacial de 30m e resolução temporal de 16 dias. Já o sensor Landsat 8 OLI foi lançado em 2013 e registra dados em 11 bandas espectrais (16 bits), com resolução espacial de $30 \mathrm{~m}$ e resolução temporal de 16 dias. As cenas utilizadas encontram-se na Figura 1 e as principais características desses três satélites, estão listadas na Tabela 1.

TABELA 1 - Principais características do Landsat 5, 7 e 8.

\begin{tabular}{|c|c|c|c|c|c|c|c|c|}
\hline \multicolumn{3}{|c|}{ Landsat 5 (TM) } & \multicolumn{3}{|c|}{ Landsat 7 (ETM +) } & \multicolumn{3}{|c|}{ Landsat 8 (OLI \& TIRS) } \\
\hline $\begin{array}{l}\text { Bandas } \\
\text { Espectr } \\
\text { ais }\end{array}$ & $\begin{array}{c}\text { Comprime } \\
\text { nto de } \\
\text { onda }(\mu \mathrm{m})\end{array}$ & $\begin{array}{c}\text { Resoluçã } \\
\text { o } \\
\text { Espacial } \\
\text { (m) }\end{array}$ & $\begin{array}{c}\text { Bandas } \\
\text { Espectr } \\
\text { ais }\end{array}$ & $\begin{array}{c}\text { Comprimen } \\
\text { to de onda } \\
(\mu \mathrm{m})\end{array}$ & $\begin{array}{c}\text { Resoluçã } \\
\text { o } \\
\text { Espacial } \\
\text { (m) }\end{array}$ & $\begin{array}{c}\text { Bandas } \\
\text { Espectr } \\
\text { ais }\end{array}$ & $\begin{array}{c}\text { Comprimen } \\
\text { to de onda } \\
(\mu \mathrm{m})\end{array}$ & $\begin{array}{c}\text { Resolução } \\
\text { Espacial (m) }\end{array}$ \\
\hline B1 & $0.45-0.52$ & 30 & B1 & 30 & $0.45-0.52$ & B1 & $0.43-0.45$ & 30 \\
\hline B2 & $0.52-0.60$ & 30 & B2 & 30 & $0.53-0.61$ & B2 & $0.45-0.51$ & 30 \\
\hline B3 & $0.63-0.69$ & 30 & B3 & 30 & $0.63-0.69$ & B3 & $0.53-0.59$ & 30 \\
\hline B4 & $0.76-0.90$ & 30 & B4 & 30 & $0.78-0.90$ & B4 & $0.63-0.67$ & 30 \\
\hline B5 & $1.55-1.75$ & 30 & B5 & 30 & $1.55-1.75$ & B5 & $0.85-0.87$ & 30 \\
\hline B6 & $10.40-12.50$ & 120 & B6 & 60 & $\begin{array}{c}10.40- \\
12.50\end{array}$ & B6 & $1.56-1.65$ & 30 \\
\hline \multirow[t]{5}{*}{ B7 } & 2.08-2.35 & 30 & B7 & 30 & $2.09-2.35$ & B7 & $2.10-2.29$ & 30 \\
\hline & & & B8 & 15 & $0.52-0.90$ & B8 & $0.50-0.67$ & 15 \\
\hline & & & & & & B9 & $1.36-1.38$ & 30 \\
\hline & & & & & & B10 & $\begin{array}{c}10.60- \\
11.19\end{array}$ & $100 *(30)$ \\
\hline & & & & & & B11 & $\begin{array}{l}11.50- \\
1251\end{array}$ & $100 *(30)$ \\
\hline
\end{tabular}

* As bandas do Sensor Infravermelho Térmico (TIRS) são adquiridas a uma resolução de $100 \mathrm{~m}$, mas são reamostradas para $30 \mathrm{~m}$ no produto de dados entregues.

FONTE: Adaptado de Teodoro e Amaral (2019).

O conjunto de dados para o município, considera 4 intervalos de tempo utilizando um algoritmo robusto no GEE capaz de integrar a aquisição de cenas dos satélites com uma porcentagem de nuvens inferior a $10 \%$. A aplicação e filtragem das cenas visa maximizar os valores de NDVI, sem sobrepor eventuais erros de resíduos (nuvens). A metodologia seguida neste trabalho, está ilustrada no fluxograma apresentado na Figura 2. 


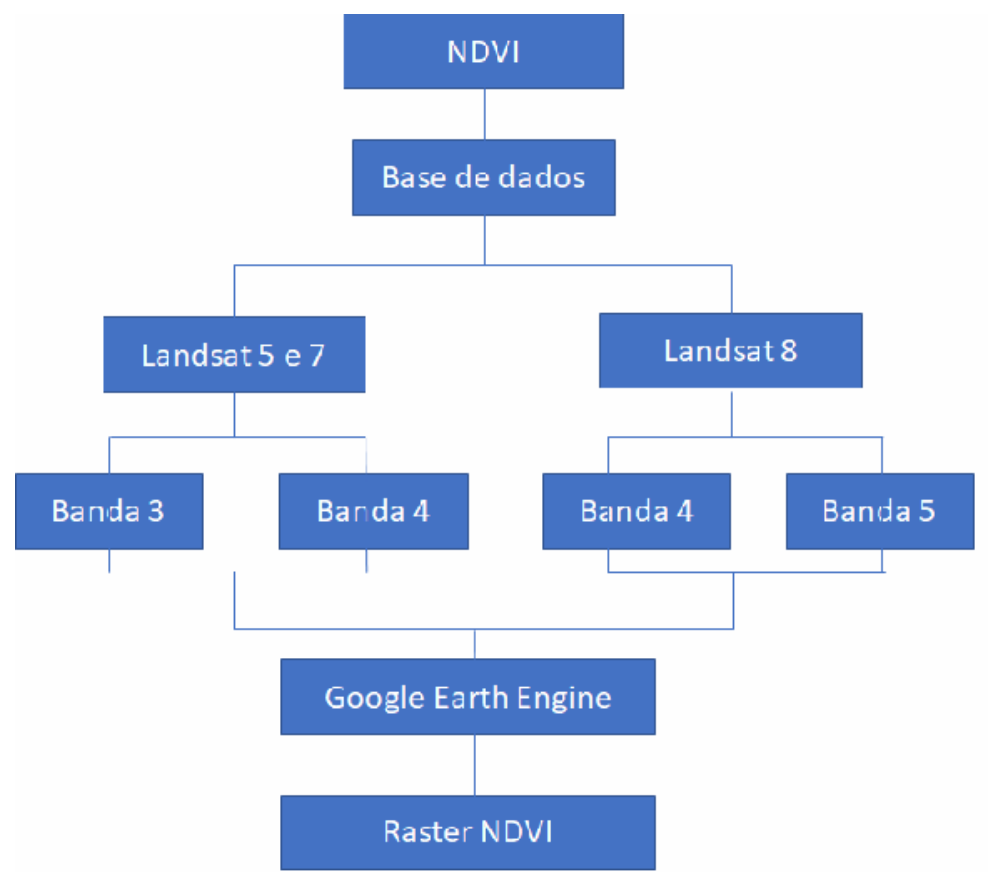

FIGURA 2 - Fluxograma representando o processo de aquisição de imagens de satélite aplicados no Google Earth Engine.

FONTE: Autores.

\section{NDVI (Normalized Difference Vegetation Index)}

O NDVI é um dos índices espectrais mais aplicados e é usado para monitorar as mudanças na vegetação sazonal e depende das propriedades de refletância da vegetação (TEODORO; AMARAL, 2019), esse índice têm a função de interpretar a condição da vegetação, seja primária ou secundária, de uma localidade (JENSEN et al., 2018), e é definido pela Equação (1):

$$
N D V /=\frac{p N R^{2}-p R E C}{p N R^{2}+p R E C}
$$

Onde, $\rho$ NIR e $\rho$ Red são, respectivamente, a reflectância da superfície da banda NIR e a reflectância da superfície da banda vermelha, este índice assume valores na faixa de -1 a 1 , onde a cobertura vegetal densa apresenta valores mais próximos a 1 e solos expostos, valores inferiores a 0,2.

\section{Mudanças do uso do solo e clima}

\section{RESULTADOS E DISCUSSÃO}

Na Figura 3, estão representados o uso e mudança do solo e variações de precipitação, temperatura máxima e mínima para o município de São Félix do Xingu. Observa-se que o uso e cobertura do solo é composto principalmente por densas florestas. Devido ao desmatamento na área ao norte e sul do município, que intensificou-se a partir de 1985, o município apresenta atualmente cerca de $30 \%$ de áreas antropizadas. É observada a maior densidade de atividades antrópicas, principalmente relacionadas à atividades da pecuária, que tem mudado significativamente o uso e cobertura do solo na região (SOUSA et al., 2017; CARVALHO et al., 2018).

Os dados de classificação apresentados na Tabela 2 fornecem um panorama de 1985, 1995, 2005 e 2015. As informações do MapBiomas, foram usadas para ajudar na caracterização da área de estudo, pois a região continha altas áreas de 
floresta em pé e alta biodiversidade, bem como a maior taxa de desmatamento do estado (VARNS et al., 2018).
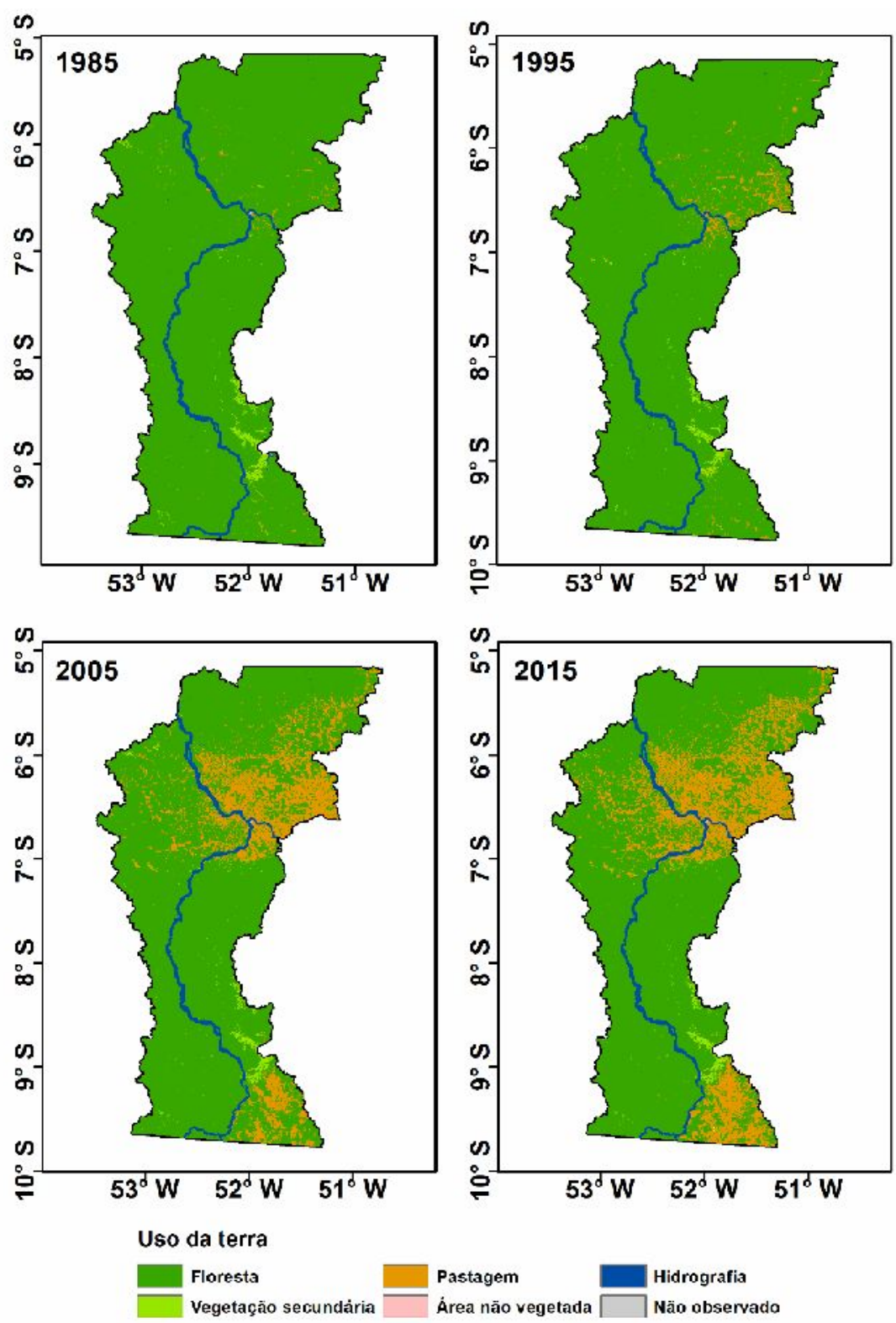

FIGURA 3 - Evolução espaço-temporal da classe de uso e cobertura da terra no município de São Félix do Xingu e variabilidade da precipitação, temperatura máxima e mínima no período de 1980 a 2015.

FONTE: Autores.

Os dados mostram 6 classes de uso e cobertura do solo, para o município de São Félix do Xingu. A classe de Floresta diminuiu em todo o período analisado, passando de $94608,10 \mathrm{~km}^{2}(96,39 \%)$ para $72915,30 \mathrm{~km}^{2}$ (74,29\%). A classe de Vegetação Secundária se manteve estável, com uma mudança de apenas 0,10\% em 2015, com o maior crescimento no ano de 2005 (1,15\%). A classe Área não vegetada, apresentou tendência de aumento, pois ocupava $9,7 \mathrm{~km}^{2}(0,01 \%)$ do município em 1985, passando a ocupar 59,8 km² (0,06\%) no ano de 2015.

Com relação à classe Pastagem, esta apresentou um aumento de 1522,50 $\mathrm{km}^{2}(1,55 \%)$ para $23215,30 \mathrm{~km}^{2}(23,65 \%)$. A maior taxa de aumento foi no ano de 
2005 e de acordo com os dados do IBGE (2017), São Félix do Xingu é o município com o maior rebanho do Brasil, com 2,22 milhões de cabeças de gado, o que poderia justificar a expansão significativa das áreas de pastagem. As práticas extensivas do sistema de gado, são utilizadas por serem mais rentáveis e por uma maior disponibilidade de terras (CAVIGLIA-HARRIS, 2018). O município também faz parte do "Arco de Fogo", que vai do nordeste ao sudoeste da Amazônia, onde há maiores ocorrências de desmatamento (SCHNEIDER; PERES, 2015).

Resultados do estudo de Schmink et al. (2019) apontam que São Félix do Xingu expandiu-se rapidamente nos anos 90, entretanto até 1992, o desmatamento ocorria fora das áreas protegidas, perto da estrada principal e longe das aldeias. No entanto, para o período de 1992 a 1999, a ocupação ocorreu em áreas anteriomente desmatadas e ao longo das estradas a oeste da região.

TABELA 2 - Distribuição das classes do MapBiomas no município de São Félix do Xingu, em quilômetros quadrado $\left(\mathrm{km}^{2}\right)$ e porcentagem (\%).

\begin{tabular}{|c|c|c|c|c|c|c|c|c|}
\hline \multirow{2}{*}{ Classes } & \multicolumn{4}{|c|}{$\mathbf{K m}^{\mathbf{2}}$} & \multicolumn{4}{|c|}{$\%$} \\
\cline { 2 - 9 } & $\mathbf{1 9 8 5}$ & $\mathbf{1 9 9 5}$ & $\mathbf{2 0 0 5}$ & $\mathbf{2 0 1 5}$ & $\mathbf{1 9 8 5}$ & $\mathbf{1 9 9 5}$ & $\mathbf{2 0 0 5}$ & $\mathbf{2 0 1 5}$ \\
\hline Floresta & 94608,10 & 92740,50 & 76822 & 72915,30 & 96,39 & 94,49 & 78,27 & 74,29 \\
\hline Vegetação & 997,60 & 571,50 & 1132,40 & 1028,10 & 1,02 & 0,58 & 1,15 & 1,05 \\
\hline Pastagem & 1522,50 & 3886 & 19232,20 & 23215,30 & 1,55 & 3,96 & 19,59 & 23,65 \\
\hline Área não vegetada & 9,70 & 9,50 & 27 & 59,80 & 0,01 & 0,01 & 0,03 & 0,06 \\
\hline Hidrografia & 1010,30 & 941 & 934,80 & 930 & 1,03 & 0,96 & 0,95 & 0,95 \\
\hline Não observado & 1,00 & 0,70 & 0,80 & 0,70 & 0,00 & 0,00 & 0,00 & 0,00 \\
\hline Total & $\mathbf{9 8 1 4 9}$ & $\mathbf{9 8 1 4 9}$ & $\mathbf{9 8 1 4 9}$ & $\mathbf{9 8 1 4 9}$ & $\mathbf{1 0 0}$ & $\mathbf{1 0 0}$ & $\mathbf{1 0 0}$ & $\mathbf{1 0 0}$ \\
\hline
\end{tabular}

FONTE: Autores.

Os dados de precipitação na Figura 4a indicam uma diminuição no quantitativo das chuvas, especialmente a partir dos anos que se acentuaram as atividades de origem antrópicas. Os picos máximos de temperatura máxima e mínima coincidem com um período de tendência positiva (mas sem significância estatística). Nota-se que há uma consistência física entre essas variáveis, pois com uma menor precipitação, espera-se maiores valores de temperatura, como observado na Figura 3. Contudo, esse resultado demonstra que somente a precipitação e temperatura não definem as variações no clima por fatores antrópicos em uma escala espacial média, resultado que pode diferir em uma escala local, onde ocorre de fato as alterações de uso e mudança da terra. 


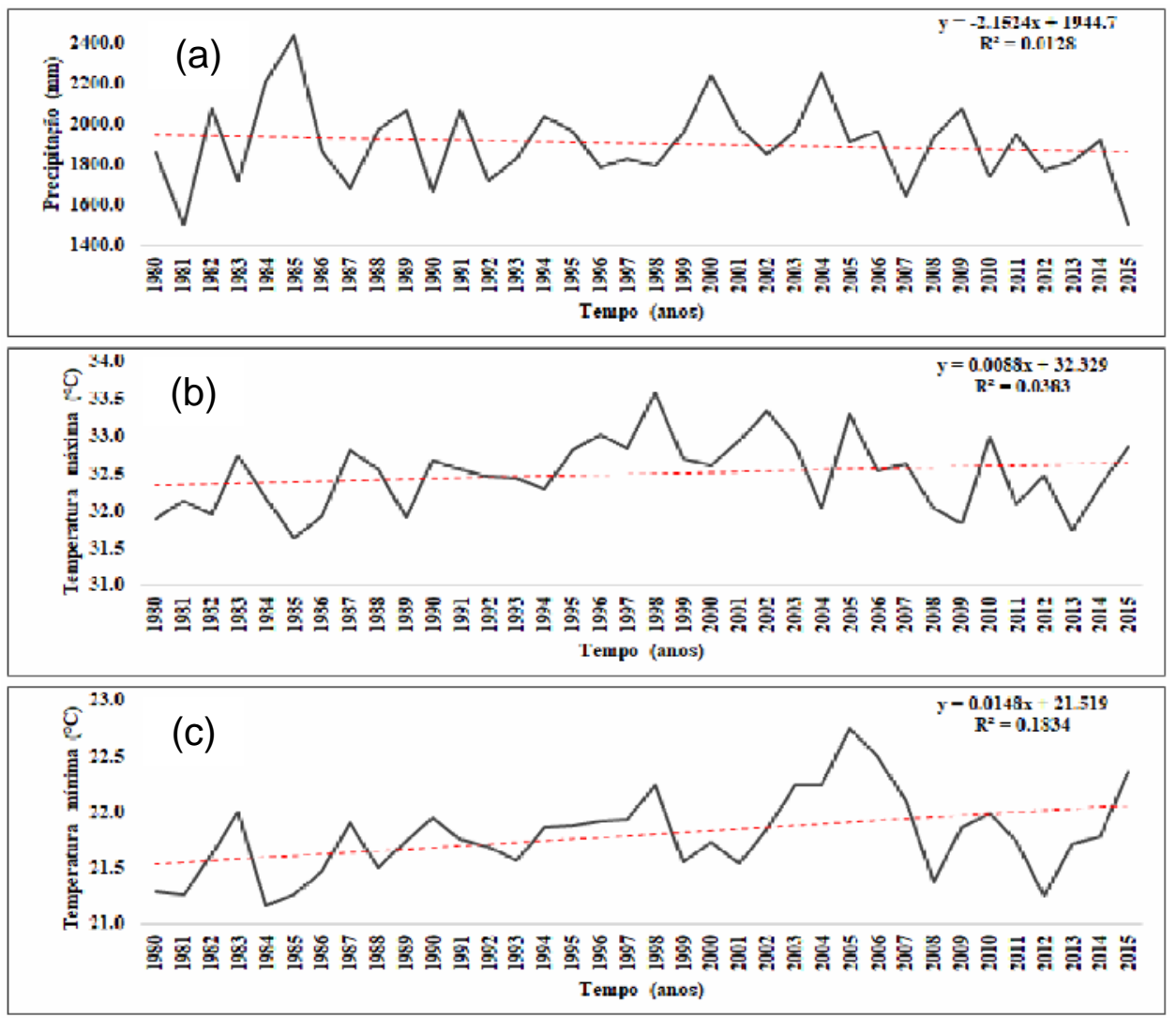

FIGURA 4 - Séries anuais de precipitação(a), temperatura máxima(b) e mínima(c) no município de São Félix do Xingu, Estado do Pará.

FONTE: Autores.

\section{Padrões espaço-temporal de NDVI}

Na Figura 5 observa-se a variabilidade do NDVI entre os períodos de 1985, 1995, 2005 e 2015. Os índices mais altos são encontrados em 1985 (Figura 5a), representado pela coloração verde intenso (NDVI > 0,6), cujo fatores antrópicos não são visíveis na região. O NDVI começa a regredir durante os anos de 1995, 2005 e principalmente em 2015 , onde os valores abaixo de 0,6 correspondem justamente as áreas de pastagem como observado nas imagens anteriores. 

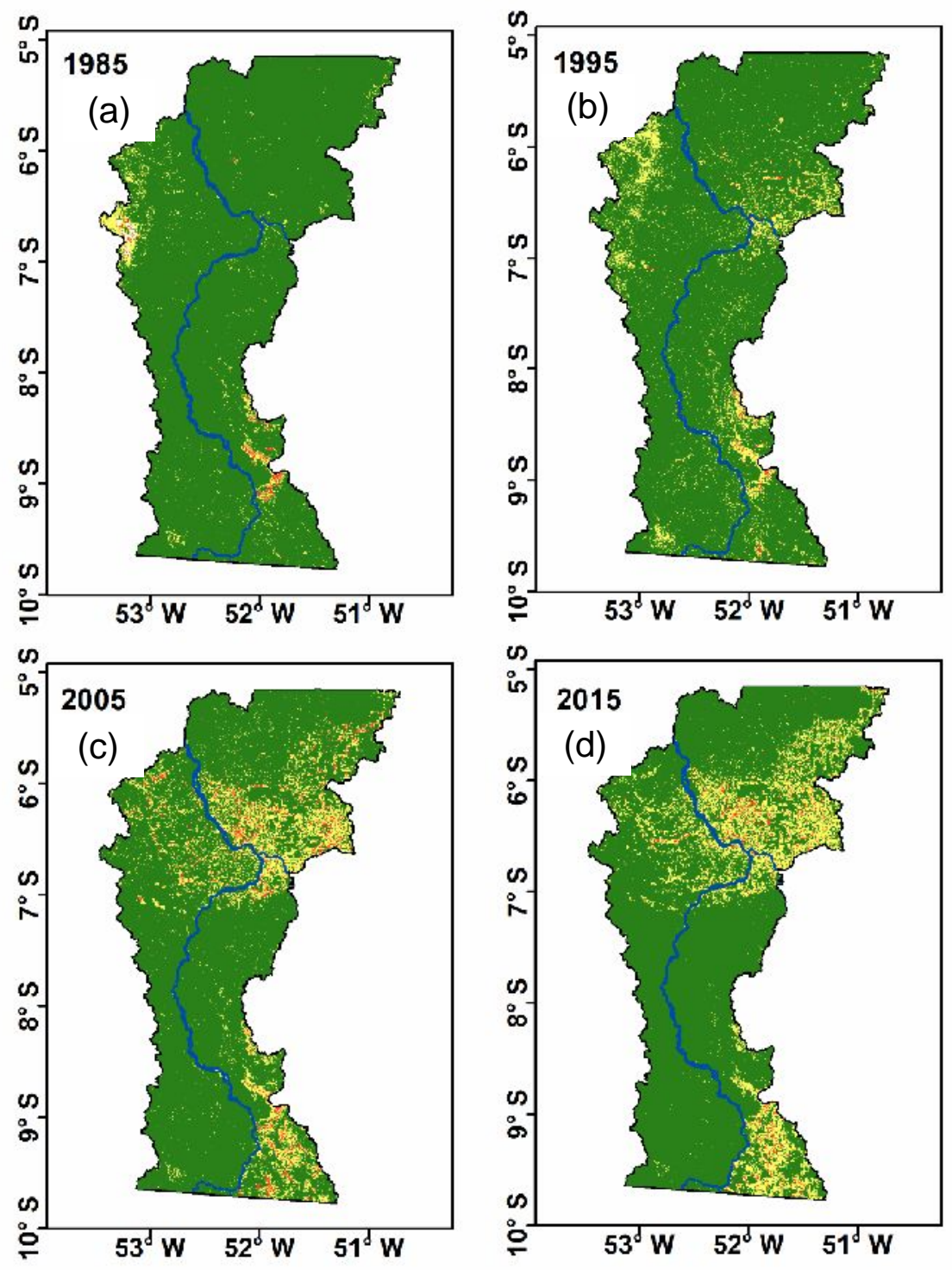

NDVI: $\square 0,0-0,2 \square 0,2-0,4 \square 0,4-0,6 \square 0,6-0,8$

FIGURA 5 - Mapa de NDVI do município de São Félix do Xingu entre os anos de (a) 1985, (b) 1995, (c) 2005 e (d) 2015.

FONTE: Autores.

\section{CONCLUSÕES}

No estudo observou-se que a mudança ocorrida no uso e cobertura da terra, está diretamente associada a fatores antropogênicos, entretanto, isto pouco contribuiu para uma variação anual significativa do clima no município de São Félix do Xingu. No entanto, a intensidade e influência das ações humanas, ocasionam uma aceleração em ritmo crescente, ocasionando uma perda da biodiversidade local e nas variações do tempo vigente associados aos mecanismos climáticos, que estão possivelmente associados durante sua propagação ao sul do Estado do Pará.

Além disso, o desmatamento oriundo das atividades de pecuária, provavelmente levou para uma alteração no ciclo hidrológico do município e das unidades de conservação que compõe o munícipio, ocasionando uma significativa redução das chuvas ao longo das décadas, explicando os picos máximos de temperaturas entre os anos de 1995 a 2015 e as alterações nos campos observados 
de NDVI. Portanto, a conservação das florestas deve ser considerada como um espaço que provê diversos serviços ecossistêmicos, para o município de São Félix do Xingu. Por essa razão, recomenda-se estudos que contemplem a avaliação dos programas e políticas públicas promovidos nas últimas décadas na região de São Félix do Xingu, a fim de identificar se estes foram capazes de gerar os benefícios ambientais relacionados com a redução do desmatamento, o melhor aproveitamento das áreas já desmatadas e na conservação dos ecossistemas locais.

\section{REFERÊNCIAS}

AYALA, L. M,; VAN EUPEN, M.; ZHANG, G.; PÉREZ-SOBA, M.; MARTORANO, L. G.; LISBOA, L. S.; BELTRAO, N. E. Impact of agricultural expansion on water footprint in the Amazon under climate change scenarios. Science of The Total Environment, v. 569, n. 570, p. 1159-1173, 2016. Disponível em: $<$ https://doi.org/10.1016/j.scitotenv.2016.06.191>. 10.1016/j.scitotenv.2016.06.191.

BOISIER, J. P.; CIAIS, P.; DUCHARNE, A.; GUIMBERTEAU, M. Projected strengthening of Amazonian dry season by constrained climate model simulations. Nature Climate Change, v. 5, n. 7, p. 656-660, 2015. Disponível em: <https://doi.org/ 10.1038/nclimate2658>. doi: 10.1038/nclimate2658.

CARVALHO, F. S.; THOMPSON, K. N. N.; LIMA, W. A. S.; SANTOS, N. F. A.; MELO, M. R. S.; SOUZA, V. Q. S.; BORGES, L. S.; GUERREIRO, A. C. Dinâmica de uso da terra, no setor agropecuário, em Paragominas - PA. Revista Agroecossistemas. v. 9, n. 2, p. 148-163, 2018. Disponível em: <https://periodicos.ufpa.br/index.php/agroecossistemas/article/view/5074>. doi: 10.18542/ragros.v9i2.5074.

CAVIGLIA-HARRIS, J. L. Agricultural innovation and climate change policy in the Brazilian Amazon: Intensification practices and the derived demand for pasture. Journal of Environmental Economics and Management, v. 90, p. 232-248, 2018. Disponível em: <https://doi.org/10.1016/j.jeem.2018.06.006>. doi: 10.1016/j.jeem.2018.06.006.

DEBORTOLI, N. S.; DUBREUIL, V.; HIROTA, M.; FILHO, S. R.; LINDOSO, D. P.; NABUCET, J. Detecting deforestation impacts in Southern Amazonia rainfall using rain gauges. International Journal of Climatology, v. 37, n. 6, p. 2889-2900, 2016. Disponível em: <https://doi.org/10.1002/joc.4886>. doi: 10.1002/joc.4886.

DEVARAJU, N.; BALA, G.; MODAK, A. Effects of large-scale deforestation on precipitation in the monsoon regions: Remote versus local effects. Proceedings of the National Academy of Sciences, v. 112, n. 11, p. 3257-3262, 2015. Disponível em: <https://doi.org/ 10.1073/pnas.1423439112>. doi: 10.1073/pnas.1423439112.

GORELICK, N.; HANCHER, M.; DIXON, M.; ILYUSHCHENKO, S.; THAU, D.; MOORE, R. Google Earth Engine: Planetary-scale geospatial analysis for everyone. Remote Sensing of Environment, v. 202, p. 18-27, 2017. Disponível em: <https://doi.org/ 10.1016/j.rse.2017.06.031>. doi: 10.1016/j.rse.2017.06.031.

GRIFFITHS, P.; JAKIMOW, B.; HOSTERT, P. Reconstructing long term annual deforestation dynamics in Pará and Mato Grosso using the Landsat archive. Remote Sensing of Environment, v. 216, p. 497-513, 2018. Disponível em: <https://doi.org/10.1016/j.rse.2018.07.010>. doi: 10.1016/j.rse.2018.07.010. 
HANSEN, M. C.; POTAPOV, P. V.; MOORE, R.; HANCHER, M.; TURUBANOVA, S. A.; TYUKAVINA, A.; ... TOWNSHEND, J. R. G. High-Resolution Global Maps of 21st-Century Forest Cover Change. Science, v. 342, n. 6160, p. 850-853, 2013. Disponível em: <https://doi.org/10.1126/science.1244693>. doi: 10.1126/science.1244693.

IBGE - Instituto Brasileiro de Geografia e Estatística, Estimativa populacional 2017. Rio de Janeiro, 2017.2 Disponível em: <http://www.ibge.gov.br/home/estatística/populacao/estimativa2017/default.shtm>. Acesso em: 11 de junho de 2019.

Disponível

Produção da Pecuária Municipal 2017. Rio de Janeiro, 2017, <https://biblioteca.ibge.gov.br/visualizacao/periodicos/84/ppm_2017_v45_br_informat ivo.pdf>. Acesso em: 12 de junho de 2019.

JENSEN, N. D.; MUDE, A. G.; BARRETT, C. B. How basis risk and spatiotemporal adverse selection influence demand for index insurance: Evidence from northern Kenya. Food Policy, v. 74, p. 172-198, 2018. Disponível em: $<$ https://doi.org/10.1016/j.foodpol.2018.01.002. doi: 10.1016/j.foodpol.2018.01.002.

JOETZJER, E.; DOUVILLE, H.; DELIRE, C.; CIAIS, P. Present-day and future Amazonian precipitation in global climate models: CMIP5 versus CMIP3. Climate Dynamics, v. $41, \quad$ n. 11 , p. 2921-2936, 2013. Disponível em: <https://doi.org/10.1007/s00382-012-1644-1>. doi: 10.1007/s00382-012-1644-1.

LAWRENCE, D.; VANDECAR, K. Effects of tropical deforestation on climate and agriculture. Nature Climate Change, v. 5, n. 1, p. 27-36, 2015. Disponível em: <https://doi.org/10.1038/nclimate2430>. doi: 10.1038/nclimate2430.

LEJEUNE, Q.; DAVIN, E. L.; GUILLOD, B. P.; SENEVIRATNE, S. I. Influence of Amazonian deforestation on the future evolution of regional surface fluxes, circulation, surface temperature and precipitation. Climate Dynamics, v. 44, n. 9, p. 2769-2786, 2014. Disponível em: <https://doi.org/10.1007/s00382-014-2203-8>. doi: 10.1007/s00382-014-2203-8.

PIELKE, R. A.; MAHMOOD, R.; MCALPINE, C. Land's complex role in climate change. Physics Today, v. 69, n. 11, p. 40-46, 2016. Disponível em: < http://www.mma.gov.br/informma/item/616-preven\%C3\%A7\%C3\%A3o-e-controledo-desmatamento-na-amaz\%C3\%B4nia>. Acesso: 27 set. 2018.

SCOTT, C. E.; MONKS, S. A.; SPRACKLEN, D. V.; ARNOLD, S. R.; FORSTER, P. M.; RAP, A.; ... WILSON, C. Impact on short-lived climate forcers increases projected warming due to deforestation. Nature Communications, v. 9, n. 1, p. 1-9, 2018. Disponível em: <https://doi.org/10.1038/s41467-017-02412-4>. doi: 10.1038/s41467-017-02412-4.

SCHNEIDER, M.; PERES, C. A. Environmental Costs of Government-Sponsored Agrarian Settlements in Brazilian Amazonia. PLOS ONE, v. 10, n. 8, p. e0134016. Disponível em: <https://doi.org/10.1371/journal.pone.0134016>. doi: 10.1371/journal.pone.0134016.

SCHMINK, M.; HOELLE, J.; GOMES, C. V. A.; THALER, G. M. From contested to 'green' frontiers in the Amazon? A long-term analysis of São Félix do Xingu, Brazil. 
The Journal of Peasant Studies, v.46, n.2, p. 377-399, 2019. Disponível em: < https://www.tandfonline.com/loi/fjps20> doi: 10.1080/03066150.2017.1381841

SEDDON, A. W. R.; MACIAS-FAURIA, M.; LONG, P. R.; BENZ, D.; WILLIS, K. J. Sensitivity of global terrestrial ecosystems to climate variability. Nature, v. $531, \mathrm{n}$. 7593, p. 229-232, 2016. Disponível em: <https://doi.org/10.1038/nature16986>. doi: 10.1038/nature16986.

SENIOR, R. A.; HILL, J. K.; GONZÁLEZ DEL PLIEGO, P.; GOODE, L. K.; EDWARDS, D. P. A pantropical analysis of the impacts of forest degradation and conversion on local temperature. Ecology and Evolution, v. 7, n. 19, p. 7897-7908, 2017. Disponível em: <https://doi.org/10.1002/ece3.3262>. doi: 10.1002/ece3.3262.

SILVA, M. J. G.; QUERINO, C. A. S.; SANTOS NETO, L. A.; MACHADO, N. G.; MILITÃO, J. S.; BIUDES, M. S. Efeito da ocupação do solo sobre o clima de Porto Velho, Rondônia, Brasil. Revista RAEGA - 0 espaço geográfico em análise. v.43, p. 232-251, 2018. Disponível em: <https://revistas.ufpr.br/raega/article/view/48753>. doi: $10.5380 /$ raega.

SOARES-FILHO, B.; RODRIGUES, H.; FOLLADOR, M. A hybrid analytical-heuristic method for calibrating land-use change models. Environmental Modelling \& Software, v. 43, p. 80-87, 2013. Disponível em: <https://doi.org/10.1016/j.envsoft.2013.01.010>. doi: 10.1016/j.envsoft.2013.01.010.

SOUSA, L. M.; ADAMI, M.; LIMA, A. M. M.; RAMOS, W. F. Avaliação do uso e cobertura da terra em Paragominas e Ulianópolis-PA, utilizando dados do projeto terraclas. Revista Brasileira de Cartografia. v. 69, n.3, p. 421-431, 2017. Disponível em: $<$ http://www.seer.ufu.br/index.php/revistabrasileiracartografia/article/view/44339/2342 1>. Acesso em: 06/04/2019.

SPRACKLEN, D. V.; GARCIA-CARRERAS, L. The impact of Amazonian deforestation on Amazon basin rainfall. Geophysical Research Letters, v. 42, n. 21, p. 9546-9552, 2015. Disponível em: <https://doi.org/10.1002/2015gl066063>. doi: 10.1002/2015gl066063.

STICKLER, C. M.; COE, M. T.; COSTA, M. H.; NEPSTAD, D. C.; MCGRATH, D. G.; DIAS, L. C. P.; ... SOARES-FILHO, B. S. Dependence of hydropower energy generation on forests in the Amazon Basin at local and regional scales. Proceedings of the National Academy of Sciences, v. 110, n. 23, p. 9601-9606, 2013. Disponível em: <https://doi.org/10.1073/pnas.1215331110>. doi: 10.1073/pnas.1215331110.

SWANN, A. L. S.; LONGO, M.; KNOX, R. G.; LEE, E.; MOORCROFT, P. R. Future deforestation in the Amazon and consequences for South American climate. Agricultural and Forest Meteorology, v. 21, n. 215, p. 12-24, 2015. Disponível em: $<$ https://doi.org/10.1016/j.agrformet.2015.07.006>.

doi: 10.1016/j.agrformet.2015.07.006.

SYKTUS, J. I.; MCALPINE, C. A. More than carbon sequestration: Biophysical climate benefits of restored savanna woodlands. Scientific Reports, v. 6, n. 1, 2016. Disponível em: <https://doi.org/10.1038/srep29194>. doi: 10.1038/srep29194. 
TEODORO, A.; AMARAL, A. A statistical and spatial analysis of Portuguese forest fires in summer 2016 considering Landsat 8 and Sentinel 2A data. Environmentals, v. $6, \quad$ n. $36, \quad$ p. 1-17, 2019. Disponível em: <https://doi.org/10.3390/environments6030036>. 10.3390/environments6030036.

VARNS, T.; CORTEZ, R.; HOVANI, L.; KINGSBURY, P. São Félix do Xingu, Brazil: a jurisdictional approach to conserving the Amazon. The Nature Conservancy, Arlington, VA, USA. Disponível em: <https:// https://www.7red.net/content/dam/tnc/nature/en/documents/TNC_Role_Jurisdictional _Programs_Sustainability_Transitions_2018.pdf>. Acesso em: 11 de junho de 2019.

XAVIER, A.C.; SCANLON, B.R.; KING, C.W. Conjunto de dados de variáveis meteorológicas diárias no Brasil (1980-2013). CLIMA Policy Brief \#2, Centro Clima/COPPE/UFRJ, Rio de Janeiro, v. 4, 2016. Disponível em: $<$ https://doi.org/10.1002/joc.4518>. doi: 10.1002/joc.4518.

ZEMP, D. C.; SCHLEUSSNER, C.-F.; BARBOSA, H. M. J.; RAMMIG, A. Deforestation effects on Amazon forest resilience. Geophysical Research Letters. v.44, p. 6182-6190, 2017. Disponível em: <https://doi.org/10.1002/2017GL072955>. doi: 10.1002/2017GL072955. 